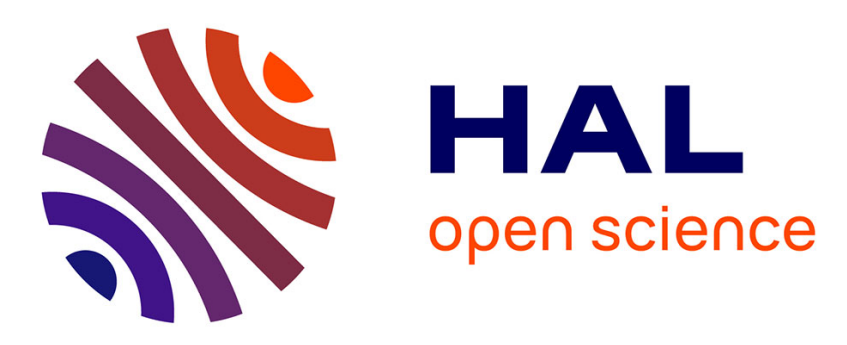

\title{
Nouvelles recherches sur l'obsidienne de Cheikh Hassan (Vallée de l'Euphrate, Syrie) au Néolithique: PPNA et PPNB ancien
}

\author{
Frédéric Abbès, Marie-Claire Cauvin, Ludovic Bellot-Gurlet, Bernard \\ Gratuze, Céline Bressy-Leandri, Gérard Poupeau
}

\section{To cite this version:}

Frédéric Abbès, Marie-Claire Cauvin, Ludovic Bellot-Gurlet, Bernard Gratuze, Céline Bressy-Leandri, et al.. Nouvelles recherches sur l'obsidienne de Cheikh Hassan (Vallée de l'Euphrate, Syrie) au Néolithique: PPNA et PPNB ancien. Syria. Archéologie, art et histoire, 2001, 78, pp.5-17. 10.3406/syria.2001.7727 . halshs-00090851

\section{HAL Id: halshs-00090851 \\ https://shs.hal.science/halshs-00090851}

Submitted on 8 Jan 2018

HAL is a multi-disciplinary open access archive for the deposit and dissemination of scientific research documents, whether they are published or not. The documents may come from teaching and research institutions in France or abroad, or from public or private research centers.
L'archive ouverte pluridisciplinaire HAL, est destinée au dépôt et à la diffusion de documents scientifiques de niveau recherche, publiés ou non, émanant des établissements d'enseignement et de recherche français ou étrangers, des laboratoires publics ou privés. 


\section{Nouvelles recherches sur l'Obsidienne de Cheikh Hassan (Vallée de l'Euphrate, Syrie) au néolithique : PPNA et PPNB Ancien} Frédéric Abbès

\section{Résumé}

L'obsidienne recueillie à Cheikh Hassan dans les occupations PPNA et PPNB ancien a été analysée chimiquement : les provenances sont diverses. Elle présente des modalités de débitage identiques à celles du silex exploité dans ce site, ce qui implique qu'elle ait été taillée par les mêmes groupes de tailleurs. Son statut et ses origines lointaines en font un matériau précieux.

\section{Abstract}

The obsidian recovered at Cheikh Hassan from the PPNA and early PPNB levels was analysed chemically. The original sources are diverse. The mode of knapping was identical to that used for flint at the site. This implies that the flint and the obsidian were worked by the same groups of knappers. Because of its status and its distant origins it must be seen as a precious material.

Citer ce document / Cite this document :

Abbès Frédéric. Nouvelles recherches sur l'Obsidienne de Cheikh Hassan (Vallée de l'Euphrate, Syrie) au néolithique : PPNA et PPNB Ancien. In: Syria. Tome 78, 2001. pp. 5-17;

doi : 10.3406/syria.2001.7727

http://www.persee.fr/doc/syria_0039-7946_2001_num_78_1_7727

Document généré le 20/04/2017 


\title{
NOUVELLES RECHERCHES SUR L'OBSIDIENNE DE CHEIKH HASSAN (VALLÉE DE L'EUPHRATE, SYRIE) AU NÉOLITHIQUE : PPNA et PPNB ANCIEN
}

\author{
Fréderic ABBÈs, Ludovic BELLOT-GURLET \\ Céline BRESSY, Marie-Claire CAUVIN \\ Bernard GRATUZE et Gérard POUPEAU
}

\begin{abstract}
Résumé - L'obsidienne recueillie à Cheikh Hassan dans les occupations PPNA et PPNB ancien a été analysée chimiquement : les provenances sont diverses. Elle présente des modalités de débitage identiques à celles du silex exploité dans ce site, ce qui implique qu'elle ait été taillée par les mêmes groupes de tailleurs. Son statut et ses origines lointaines en font un matériau précieux.
\end{abstract}

Abstract - New research on the obsidian of Cheikh Hassan (Euphrates'valley, Syria) at the Neolithic :

PPNA and early PPNB

The obsidian recovered at Cheikh Hassan from the PPNA and early PPNB levels was analysed chemically. The original sources are diverse. The mode of knapping was identical to that used for flint at the site. This implies that the flint and the obsidian were worked by the same groups of knappers. Because of its status and its distant origins it must be seen as a precious material.

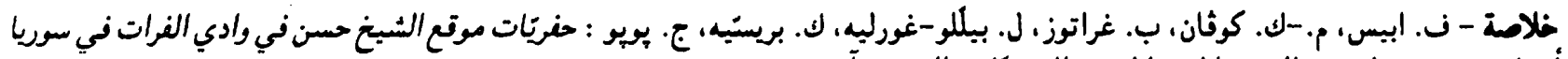

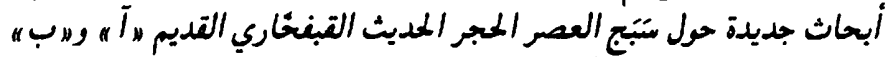

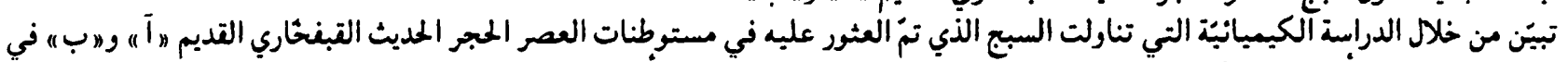

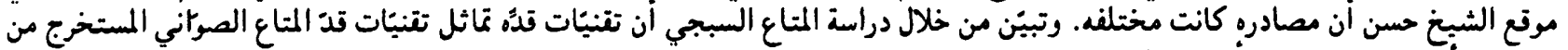

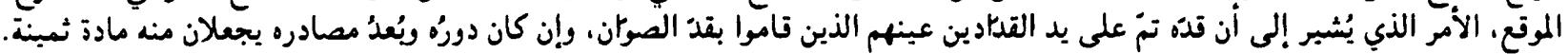

Cheikh Hassan est un tell, naguère situé sur la rive gauche de l'Euphrate (fig. 1), mais disparu depuis la mise en eau du barrage de Tabqa. Deux campagnes y ont été pratiquées : l'une en 1976, l'autre en 1993; dans l'intervalle de temps les

F. ABBìs : Institut français d'archéologie du Proche-Orient, BP 3694, Damas, Syrie.

L. BELLOT-GURLET : CNRS-UMR 7075, Laboratoire de Dynamique. Interraction et Réactivité. 2 rue Henri Dunant, 94320 Thiais.

C. BRESSY : CNRS-UMR 6636, Économies, sociétés et environnements préhistoriques, Processus Culturels, Biologiques et Environnements quaternaires, Institut Dolomieu, Université Joseph Fourier, 15 rue Maurice Gignoux, 38031 Grenoble; CNRS-UMR 5025, Groupe de Géophysique nucléaire, Laboratoire de Géodynamique des Chaînes Alpines, Université Joseph séparant, le niveau de l'Euphrate était monté de façon telle qu'il a empêché les recherches concernant le plus ancien Néolithique (PPNA). La campagne de 1993 a concerné des niveaux plus récents (PPNB) avant la totale immersion du site.

Fourier, Maison des Géosciences. BP 53, 38041 Grenoble Cedex.

M.-C. Cauvin : CNRS-UMR 5647, Institut de Préhistoire orientale. Jalès, 07460 Berrias.

B. Gratuze : CNRS-UMR 5060, Centre Ernest Babelon, Institut de Recherche sur les Archéomatériaux, 3D rue de la Férollerie, 45071 Orléans Cedex 2.

G. POUPEAU : CNRS-UMR 5060, Centre de Recherche en Physique appliquée à l'Archéologie. Institut de Recherche sur les Archéomatériaux, Maison de l'Archéologie, 33607 Pessac. 


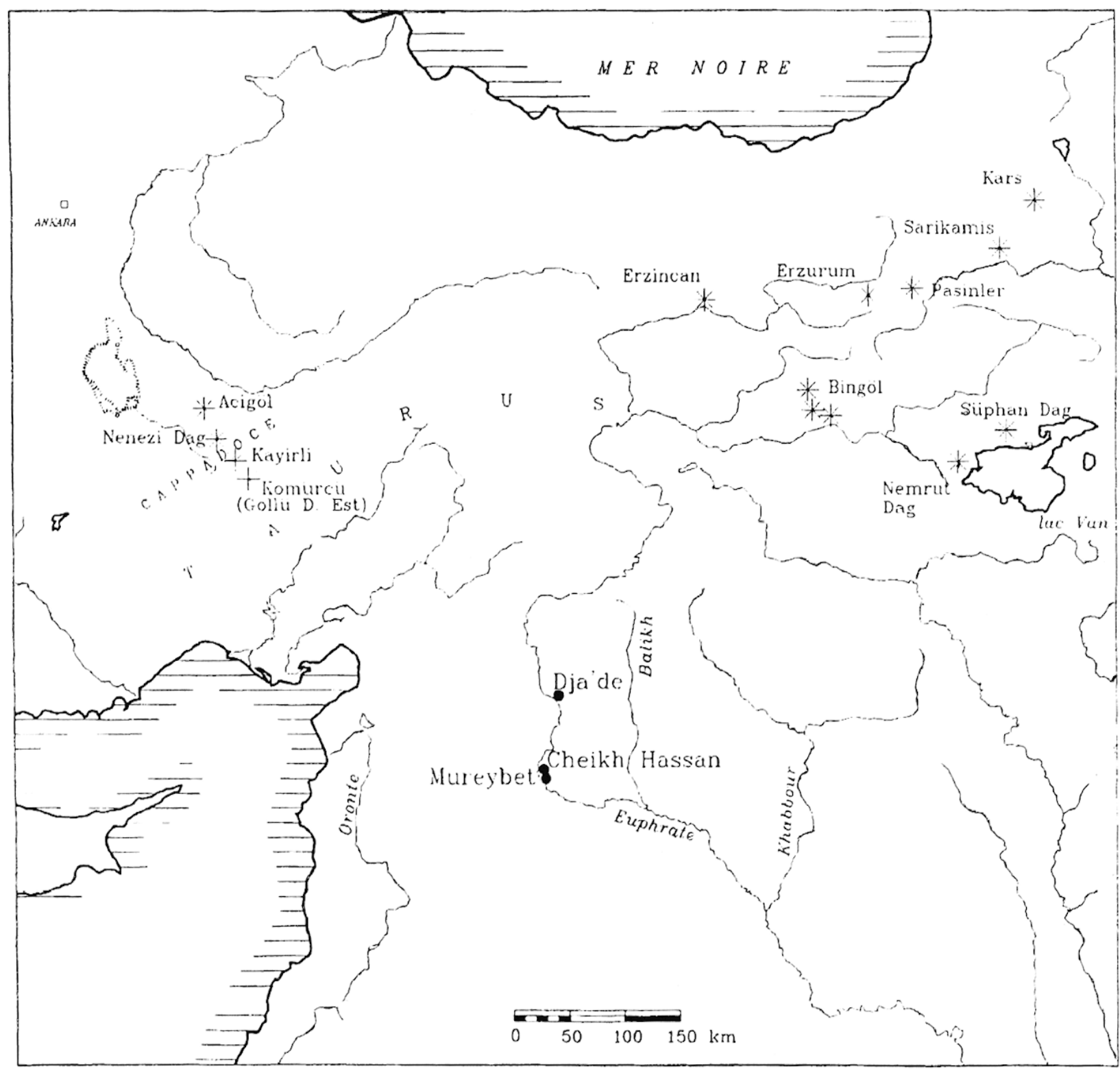

Fig. 1. - Carte des gisements archéologiques (indiqués par des cercles) et des sources d'obsidienne (notées par des étoiles).

\section{CAMPAGNE DE 1976 PPNA FINAL}

Une campagne de sondages, entreprise en 1976 sous la direction de J. Cauvin, a mis en évidence dans quatre d'entre eux la fin de la culture Mureybétienne qui correspond, à Mureybet même, à la phase IIIB'. Il s'agit de la fin du PPNA ou autrement dit de la fin de la "période 2 » telle qu'elle a été définie à la Maison de l'Orient ${ }^{2}$. Ces niveaux sont datables

I. J. Cauvin 1980, 1994.

2. Hours et al. 1994. d'environ 9500 B.P. soit environ $8700 \mathrm{cal}$. B.C. Il s'agit ici de sédentaires, pourvus notamment d'une architecture rectangulaire.

\section{Le matériel}

L'industrie lithique taillée est essentiellement en sile $x^{3}$ mais on trouve néanmoins, assez rare, du matériel en obsidienne ${ }^{4}$ éparpillé dans les différents sondages, l'un d'entre eux étant un peu plus récent (LO) que les autres (DS, LB, MC).

3. ABBĖS 1993.

4. M.-C. Cnuvin, Chataigner 1998. 
Il y a en tout 65 artefacts sous forme de :

- fragments de lames (lames de plein débitage : fig. $2, \mathrm{n}^{0 \mathrm{~S}} 1,3,4,6,11-13$ et lames techniques, débordantes sur crête frontale : fig. $2, \mathrm{n}^{0 \mathrm{~s}} 8,14$ );

- quelques éclats dont une ouverture de plan de frappe (fig. $2, \mathrm{n}^{\circ} 2$ );

- esquilles.

\section{Le débitage}

La présence de l'ensemble de ces éléments est révélatrice d'un débitage sur place de ce matériau. Le débitage se rattache à une production de type bipolaire comparable en partie à celle réalisée sur le silex fin. Le recours à la pression semble exclu. La faiblesse de l'échantillon ne permet pas de déterminer plus précisément le type même de production bipolaire, réalisé sur obsidienne : la majorité des éléments retrouvés peuvent en effet être rattachés à plusieurs types de débitage. Ils comportent en effet des lames elles-mêmes unipolaires ou bipolaires qui rentrent toutes dans les schémas de chaînes opératoires reconnues ${ }^{5}$. Néanmoins, l'existence de quelques fragments de grandes lames (fig. $2, n^{\circ} 16$ ) soulève le problème de leur obtention. Plusieurs cas de figure sont envisagés : il peut s'agir de grandes lames de correction des surfaces de débitage des nucléus, comme cela est attesté pour les débitages sur silex de ce même niveau de Cheikh Hassan, ou bien d'une production particulière en clle-même, distincte de celle des petites lames plus généralement retrouvées. Les deux options sont envisageables et parfaitement connues ailleurs pour ces périodes ${ }^{6}$.

L'étroitesse de l'éclat à crête d'ouverture de plan de frappe retrouvé (fig. $2, n^{\circ} 2$ ) évoque des exemplaires similaires sur silex du site voisin de Mureybet, mais beaucoup plus récents puisqu'ils y appartiennent au PPNB moyen. À Mureybet, ces ouvertures de plan de frappe sont associées à des nucléus naviformes étroits, orientés vers la production de lames préfaçonnées. On n'ose en revanche, faute de documents suffisants, tirer de conclusion de l'unique exemplaire de Cheikh Hassan.

Enfin, un fragment de grande lame à troncature abrupte proximale (fig. $2, n^{0} 5$ ) mérite une description particulière. La troncature a servi de plan de frappe ou de pression à des enlèvements lamellaires en direction de la surface supérieure de la lame. Une des lamelles est restée en partie accrochée à la lame. Cette technique évoque celle décrite par Perlès et Pélegrin ${ }^{7}$ pour l'obtention de lamelles dites d'Orville. Cette pièce peut donc être considérée soit comme un nucléus à lamelles (ces dernières faisant défaut sur le site), soit comme un témoignage d'un procédé d'amincissement du support. Les enlèvements lamellaires seraient alors une véritable retouche préfigurant les retouches plates en pelure, typiques à partir du PPNB moyen de l'Euphrate. De plus, on relève la présence d'une pièce esquillée (fig. $2, n^{\circ} 8$ ) évoquant celles qu'on trouvera, plus nombreuses, un peu plus tard, plus en amont sur l'Euphrate, dans le PPNB ancien à Dja'de, également en obsidienne ${ }^{8}$.

La présence d'armatures de projectiles en obsidienne est indiscutable (fig. $2, \mathrm{n}^{\text {os }} 9,10,12-14$, 16). Elles sont obtenues à partir de lames de plein débitage $\left(\mathrm{n}^{0 \mathrm{~s}} 12,13\right)$ ou de lames techniques $\left(\mathrm{n}^{\circ} 14\right)$. Leur base est aménagée soit par troncature, selon des modalités présentes uniquement sur ce type d'artefact, soit par pédoncule. Le pédoncule est alors peu épais, court et obtenu par retouches abruptes directes $\left(\mathrm{n}^{0 \mathrm{~S}} 13-14\right)$. Ces pointes sont nombreuses en silex à Mureybet III. Elles sont à Cheikh Hassan également présentes et même abondantes en silex $\left(n^{\circ} 17\right)$. Des armatures à base tronquée en obsidienne $\left(\mathrm{n}^{\text {os }} 12,16\right)$, semblables elles aussi à celles en silex $\left(n^{\circ} 15\right)$, portent à leur extrémité proximale des retouches bifaciales obliques. L'une d'elles $\left(n^{\circ} 12\right)$ porte le témoignage de son utilisation en armature offensive sous la forme d'un "coup de burin " dû à l'impact à son extrémité distale. À ces pièces s'ajoutent des fragments proximaux (troncature, fig. $2, n^{\circ} 6$, ou pédoncule, $\left.n^{\circ} 10\right)$ ou distaux $\left(n^{\circ} 9\right)$. Outre les lames et éclats retouchés, il y a également une lame pédonculée à cassure distale accidentelle et deux paires d'encoches latérales $\left(\mathrm{n}^{\circ} 11\right)$ : clle pourrait avoir aussi servi de projectile.

Ainsi, on constate que ce matériau d'obsidienne a été débité et retouché sur place selon les mêmes modalités que le silex. Si on le compare plus précisément à celui en silex pour un des sondages (DS) où les deux matériaux ont été étudiés, on constate à quel point l'obsidienne est peu abondante : il n'y a aucun nucléus alors qu'il y en a 48 en silex; seulement 6 éclats (fig. $2, n^{\circ} 2$ ) en obsidienne contre 1417 (soit $0,4 \%$ ), 7 lames 

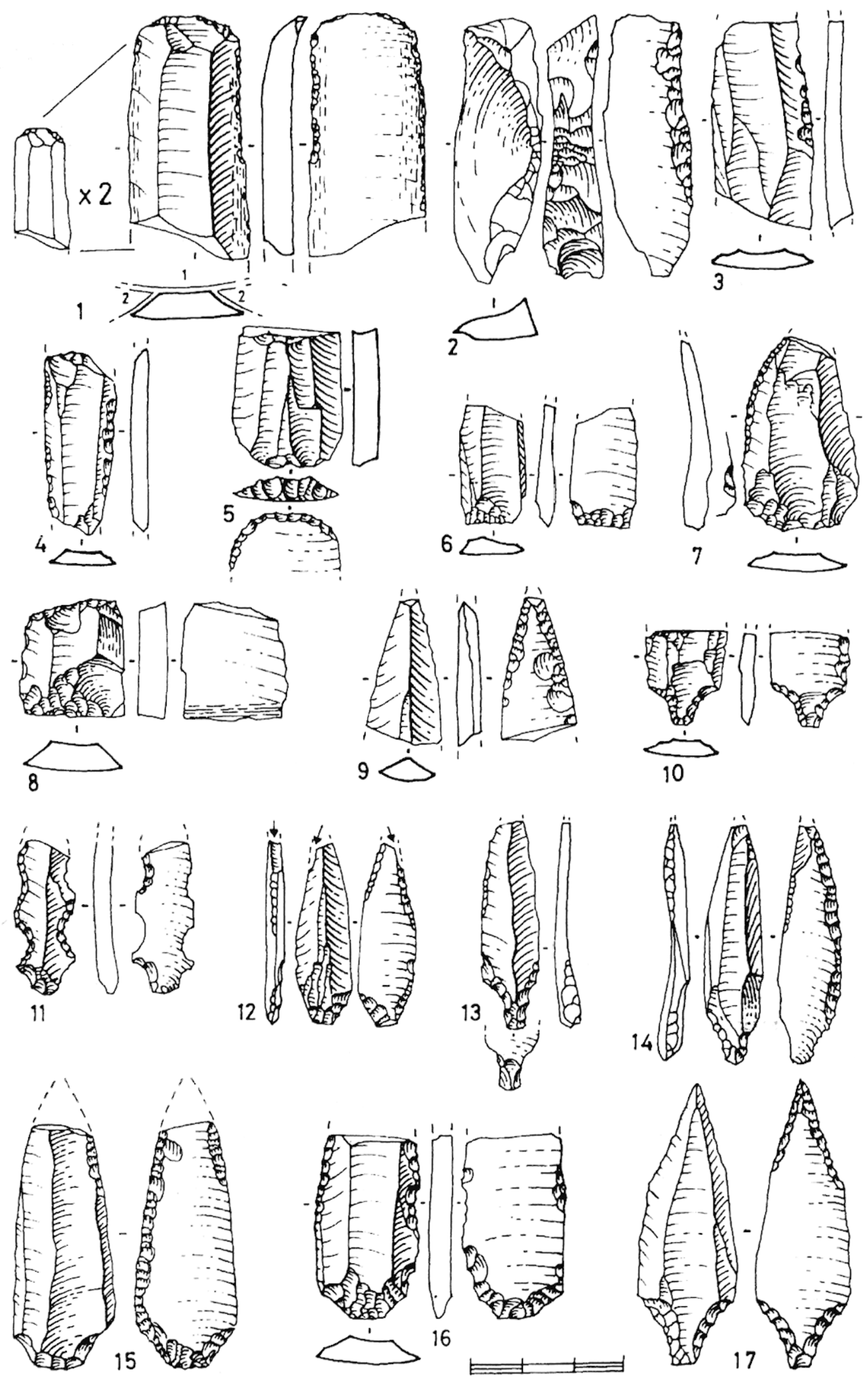

Fig. 2. - Cheikh Hassan : Mobilier PPNA en obsidienne (sauf les $\mathrm{n}^{0 \mathrm{~s}} 15$ et 17 en silex) provenant des secteurs MC $: \mathrm{n}^{\text {os }} 1,4,12,14 ; \mathrm{DS}: \mathrm{n}^{\mathrm{OS}} 2,3,11,13,15,17 ; \mathrm{LB}: \mathrm{n}^{\mathrm{os}} 5-8: \mathrm{LO}: \mathrm{n}^{\mathrm{OS}} 9,10,16$. 
$\left(\mathrm{n}^{\mathrm{os}} 3,11\right.$ ) contre 1009 (soit $0,7 \%$ ). Quant aux armatures de projectiles de ce secteur, il y en a 1 contre 51 en silex ( $2 \%)$. L'obsidienne est donc certes rare et à la différence du silex, elle n'est pas d'origine locale.

\section{Détermination de provenance}

\section{Les analyses physico-chimiques}

Les recherches concernant la provenance de cette matière première ont été effectuées par des analyses physico-chimiques.

En effet, l'obsidienne est une roche vitreuse d'origine volcanique issue d'un refroidissement très rapide d'une lave acide dont la composition est normalement caractéristique de chaque éruption. Ainsi, le gîte naturel dont provient le matériau peut-il être très bien défini sous l'angle de sa composition chimique.

Plusieurs campagnes d'analyses ont été effectuées dans différents laboratoires :

- une première série de mesures (huit échantillons) à la fin des années quatre-vingt en activation neutronique à Bradford par S.E. Warren (seules les déterminations de provenance ont fait l'objet d'une communication personnelle à M.-C. Cauvin ${ }^{9}$ );

- trois autres échantillons provenant du secteur LO ont été étudiés par activation avec des neutrons rapides de cyclotron (ANRC) puis par spectrométrie de masse à torche à plasma couplée à l'ablation laser (LA-ICP-MS) à Orléans ${ }^{10}$;

- une troisième série d'analyses de trois échantillons tous transparents $\left(\mathrm{n}^{\text {os }} 1\right.$ à 3 ), provenant là aussi du secteur LO, a été pratiquée à Grenoble par spectrométrie d'émission atomique (ICP-AES, pour les éléments majeurs) et spectrométric de masse (ICP-MS, pour les éléments traces) associées à une torche à plasma" ${ }^{\text {; }}$;

- plus récemment enfin, cinq échantillons (deux opaques, $\mathrm{CH} 76-4$ et 5 ; deux transparents, $\mathrm{CH}$ 76-7 et 8 ; un vert, $\mathrm{CH}$ 76-6) provenant des secteurs les plus anciens (MC/LB/DS) ont été analysés dans le même laboratoire et selon les mêmes procédures ${ }^{12}$.

L'ensemble des mesures disponibles sur les pièces de Cheikh Hassan est explicité de façon détaillée dans le tableau (fig. 3). Certaines ont déjà fait l'objet de publications (voir les notes du tableau), d'autres sont originales. Le tableau présente, regroupés par laboratoire, les échantillons analysés avec les teneurs de chacun des éléments majeurs et traces à partir desquels sont établies les provenances et définies les grandes familles d'obsidiennes (calco- ou per- alcaline, en fonction de teneurs relatives en $\mathrm{Al}_{2} \mathrm{O}_{3}, \mathrm{Na}_{2} \mathrm{O}, \mathrm{K}_{2} \mathrm{O}, \mathrm{CaO}$ ). Les figures 4 et 5 présentent des diagrammes illustrant les principales caractéristiques des compositions chimiques des obsidiennes de Cheikh Hassan.

\section{Les résultats}

Les résultats montrent que 12 des 19 échantillons proviennent d'Anatolie centrale, du sud de la Cappadoce, et plus précisément du Göllü Dag Est, c'est-à-dire principalement des affleurements de Kayirli Est ou de Kömürcü ${ }^{13}$, où l'obsidienne est souvent assez transparente, comme l'est notamment la lamelle $n^{\circ} 1$ de la figure 2 . Sur la base des caractérisations compositionnelles disponibles sur des échantillons des affleurements de Kayirli Est et de Kömürcü, il est actuellement impossible de discriminer les obsidiennes de ces deux secteurs du Göllü Dag. Parmi les douze exemplaires nous trouvons :

- cinq échantillons attribués par Warren au "groupe B1 », que l'on rattache à présent au Göllü Dag Est;

- un échantillon transparent (fig. $2, n^{\circ} 10$ ) attribué par Gratuze au « groupe 3 » $^{14}$, identifié depuis au Göllü Dag Est;

- six échantillons analysés à Grenoble. Ils correspondent aux exemplaires $\mathrm{CH}$ 76-5 (opaque) et CH 76-1, 2, 3, 7, 8 (transparents).
9. M.-C. CAUVIN 1991.

10. Gratuze et al. 1993; Gratuze 1999.

11. MARTINETTO 1996.
12. Bellot-GurLet 1998.

13. POIDEVIN 1998.

14. GRATUZE et al. 1993. 


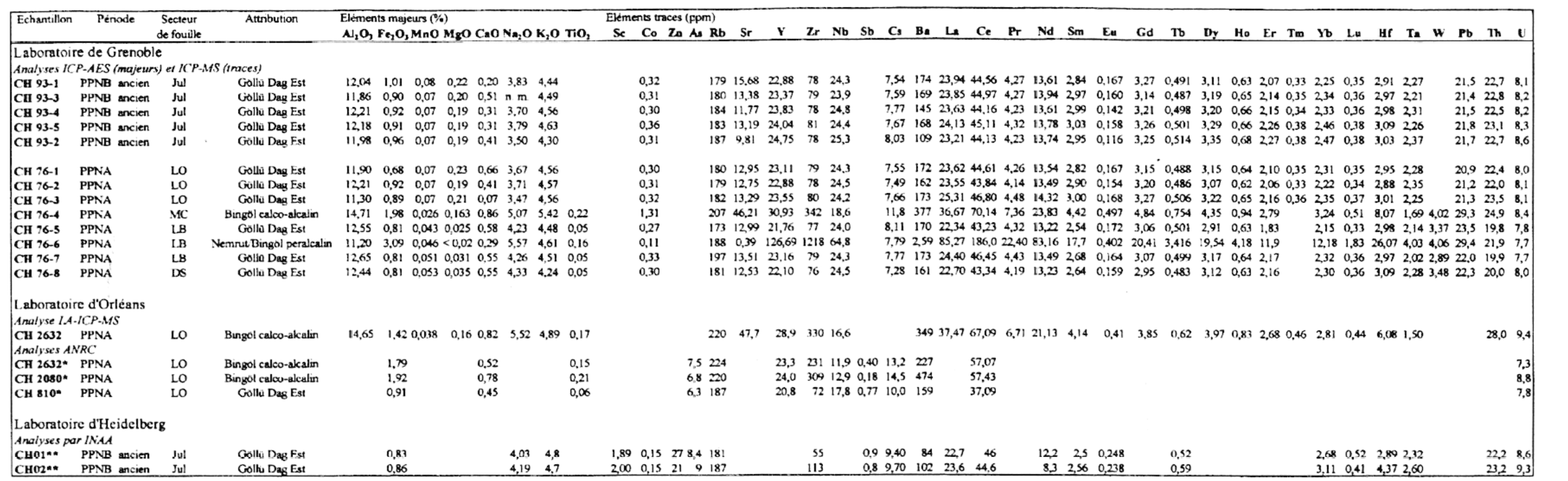

NB : Les valeurs obtenues par ANRC à Orléans sont à l'origine calculées sous forme de rapport au sodium (GRATUZE et al. 1993). Afin de permettre une comparaison plus facile de l'ensemble des valeurs, des teneurs corrigées ont été recalculées. Les valeurs utilisées pour le calcul sont les concentrations moyennes en sodium de chacun des groupes, elles sont de $3,63 \%\left(4,9 \% \mathrm{Na}_{2} \mathrm{O}\right)$ pour les obsidiennes calco-alcalines de Bingöl et de $2,97 \%$ ( $4 \% \mathrm{Na}_{2} \mathrm{O}$ ) pour celles du Göllü Dag Est.

* Données utilisées dans Gratuze et al. 1993 pour la définition des compositions moyennes des groupes compositionnels.

** Données publiées dans PernicKa et al. 1997

Fig. 3. - Présentation des compositions élémentaires des obsidiennes de Cheikh Hassan. 

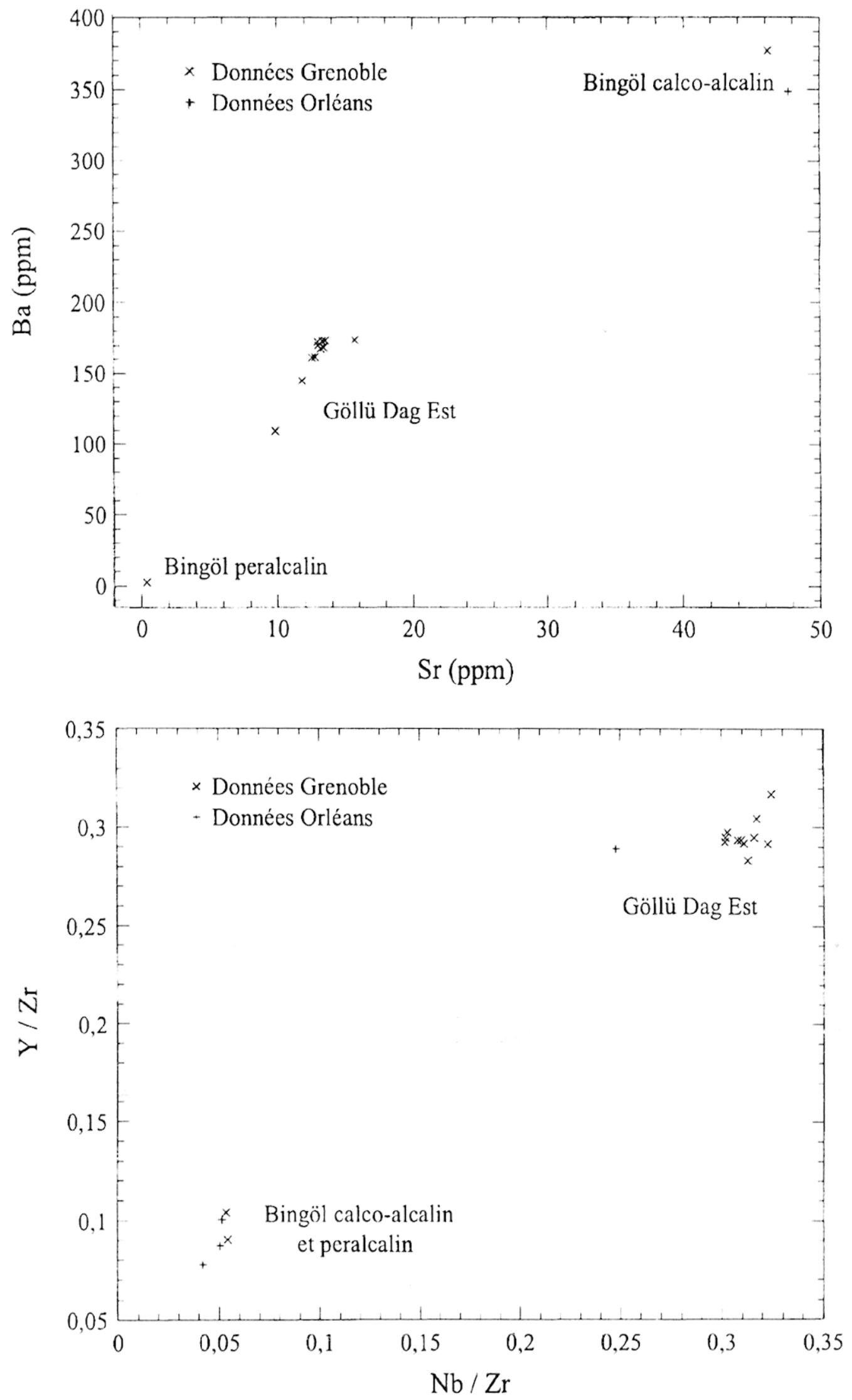

Fig. 4. - Diagrammes binaires Ba vs. Sr et $\mathrm{Y} / \mathrm{Zr}$ vs. $\mathrm{Nb} / \mathrm{Zr}$ illustrant les discriminations entre artefacts de différentes provenances. 


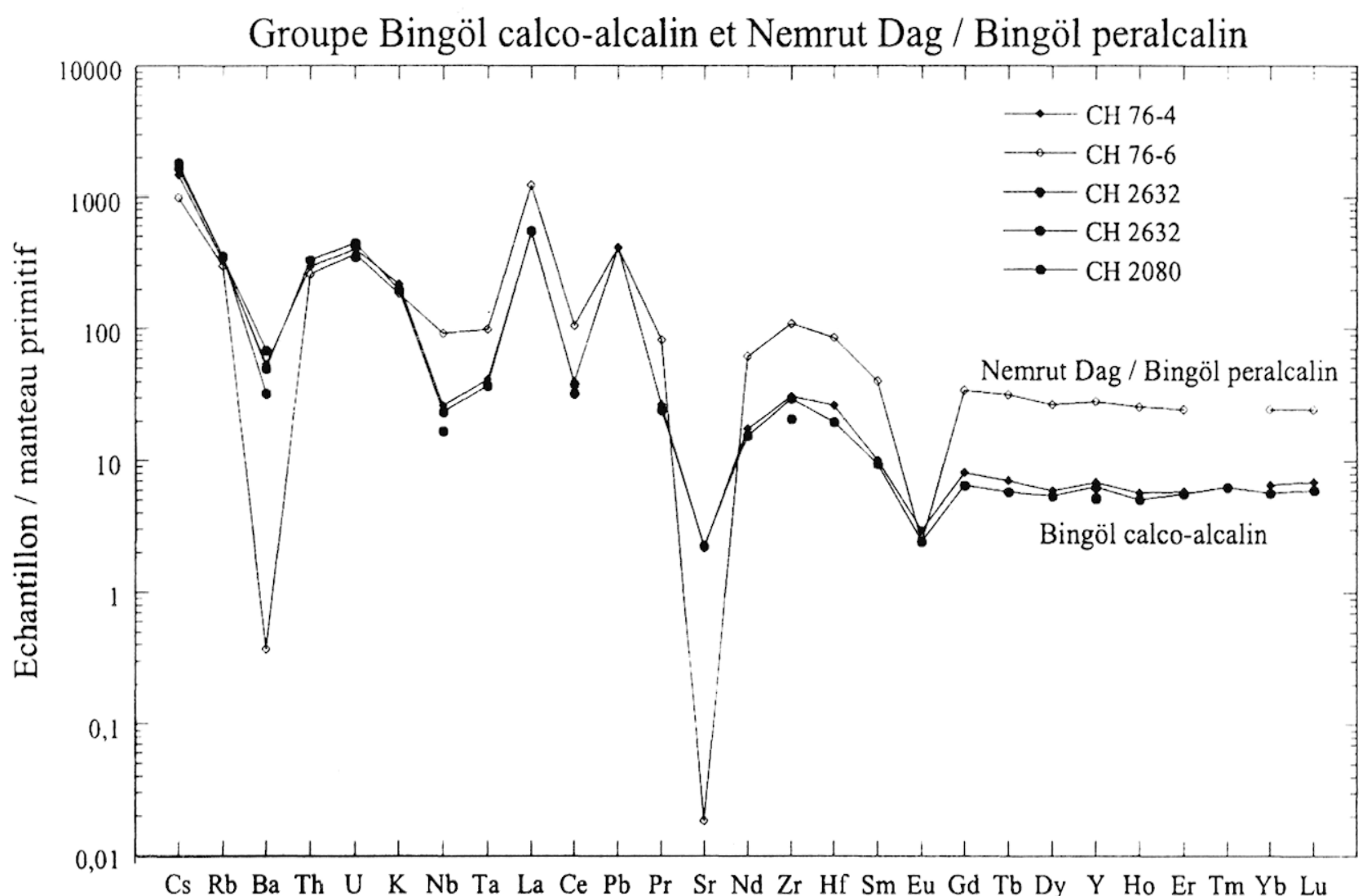

Groupe Göllü Dag Est

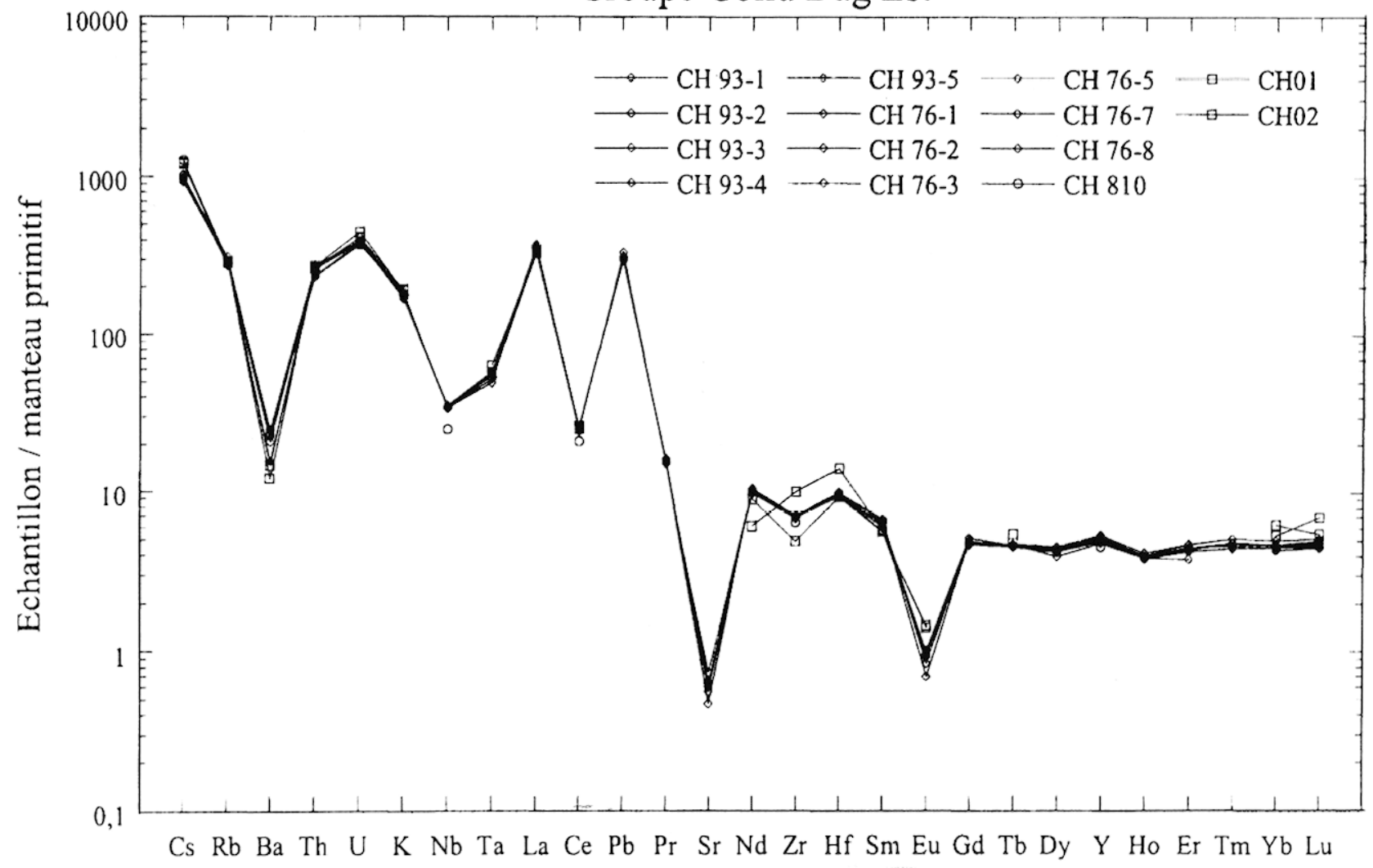

Fig. 5. - Représentation des différentes signatures compositionnelles en utilisant des diagrammes de normalisation au manteau primitif (valeurs de SUN et MCDONOUGH 1989). 
Les sept autres échantillons proviennent d'Anatolie orientale : six d'entre eux présentent une composition calco-alcaline attribuable de façon certaine par les divers auteurs à la région de Bingöl. Ils se répartissent ainsi :

- trois échantillons attribués par Warren à son "groupe B2 »;

- deux échantillons opaques attribués par Gratuze, l'un à son "groupe 2 " (fig. 2, ${ }^{\circ} 9$ ), l'autre à son " groupe 4 "- provenance non identifiée avec les analyses par ANRC en 1993, puis tous réattribués au seul groupe calco-alcalin de Bingöl avec les analyses par LA-ICP-MS en 1999;

- un échantillon opaque, analysé à Grenoble, $(\mathrm{CH}$ 76-4);

- le septième échantillon analysé à Grenoble $(\mathrm{CH}$ 76-6), le seul d'aspect verdâtre à l'œil nu, a une composition peralcaline. Actuellement, sur la base des analyses chimiques réalisées, il est difficile d'attribuer sans équivoque certaines obsidiennes peralcalines de la région à l'une ou l'autre des sources de Bingöl ou du Nemrut Dag. C'est le cas de cette pièce.

Les sources de Bingöl sont aisément accessibles par la vallée de l'Euphrate et ses affluents. L'éventuelle provenance du Nemrut Dag d'une pièce ferait envisager un autre itinéraire utilisant la haute vallée du Tigre. Ces liens entre les sites archéologiques de la moyenne vallée de l'Euphrate et le Nemrut Dag seront par exemple soulignés par la présence certaine d'une obsidienne du Nemrut Dag au PPNB ancien de Dja'de et d'autres au PPNB moyen de Mureybet et de Halula ${ }^{15}$. Quant au Göllü Dag il est beaucoup plus éloigné que l'Anatolie orientale de Cheikh Hassan. Cependant, le lien avec le nord-ouest et plus précisément la zone à ophiolites du Taurus est aussi confirmé par les études de provenance d'autres matériaux ayant été utilisés pour la parure ${ }^{16}$.

Ainsi, ce mobilier en obsidienne, quoique rare, présente une réelle diversité d'origine quant au matériau lui-même. En effet, si l'obsidienne cappadocienne du Göllü Dag Est est présente au Levant dès le Natoufien, celle de l'Anatolie orientale n'y apparaît qu'à la fin du PPNA ici même comme à Mureybet. En revanche, les techniques utilisées pour débiter la matière première ainsi que la retouche sont identiques à celles employées pour le silex et donc suggèrent l'intervention soit des mêmes personnes soit de tailleurs appartenant à la même tradition au niveau de la fabrication. Les lames, les flèches sont de dimensions moindres, en général, que celles en silex, le matériau de départ devant être exploité au maximum. Enfin on note que cette obsidienne, qui a dû, même à l'état brut, attirer le regard, a été utilisée surtout pour des armatures de flèches et que l'une d'elles (fig. 2 . $n^{\circ} 12$ ), celle qui porte l'impact de frappe, a été recueillie dans une tombe près d'un crâne.

\section{CAMPAGNE DE 1993 PPNB ANCIEN}

Cette campagne de fouilles, sous la direction de D. Stordeur ${ }^{17}$, a permis de dégager sur $125 \mathrm{~m}^{2}$ des niveaux plus récents que ceux que nous venons de décrire. Ils correspondent au PPNB ancien c'est-àdire à la phase IVA de Mureybet, ou plus généralement, au tout début de la "période 3 " décrite à la Maison de l'Orient. À Dja'de, cette occupation est datée de 9200 à 9100 B.P., soit autour de $8400 \mathrm{cal}$. B.C.

\section{Le matériel}

L'industrie lithique taillée en silex est toujours très abondante. Celle qui provient de deux secteurs particuliers a été analysée en détail ${ }^{18}$ :

- secteur atelier (Jul) : il s'agit du mobilier provenant d'un espace considéré comme spécialisé dans la taille du silex;

- secteur habitat (FK) : il comporte des architectures.

Le matériel en obsidienne y est très rare ( 45 artefacts):

- il se répartit principalement dans l'espace spécialisé pour la taille ( 22 ex.);

- 13 exemplaires seulement ont été trouvés dans l'aire d'habitat. Notons cependant qu'il y a un nucléus dans chaque zone;

- les 10 autres pièces étaient éparpillées dans les autres secteurs de la fouille.

Si on compare les 35 pièces en obsidienne à celles en silex provenant de ces deux secteurs particuliers (Jul ou JL et FK), on constate qu'elles correspondent à $0,7 \%$ des artefacts taillés dans l'ensemble des deux matériaux (silex et obsidienne), et plus précisément que les nucléus 
d'obsidienne représentent 5,6\% du total des nucléus, les lames $2 \%$, les éclats et esquilles $0,4 \%$. Le matériel en obsidienne se compose aussi de :

- quelques éclats de façonnage, de nucléus (fig. 6, $\left.\mathrm{n}^{\text {os }} 2,10\right)$ avec un éclat extrait depuis une crête $\left(n^{\circ} 6\right)$;

- lames techniques, lames d'entretien de surface de débitage $\left(n^{\text {os }} 4,11\right)$;

- lames débordantes $\left(\mathrm{n}^{\text {OS }} 3,9\right)$;

- esquilles;

- quelques fragments de lames de plein débitage aussi $\left(n^{\circ} 8\right)$.

\section{Le débitage}

Ici non plus, aucune des pièces retrouvées ne semble pouvoir être attribuée à un débitage par pression. Tous les stigmates de débitage identifiables se rapportent en effet à un débitage à la percussion directe sur nucléus bipolaire ou naviforme. Les très rares éléments laminaires retrouvés s'intègrent dans la variabilité des lames produites par ces nucléus. Il est possible également qu'une exploitation lamellaire ait eu lieu sur le site. Ceci est peut-être suggéré par la présence d'un fragment de nucléus ou de gros éclat (?) montrant des tentatives d'enlèvements lamellaires mais aucune vraie lamelle sur obsidienne n'a cependant pu être identifiée sur le site.

Du reste tous les outils, comme les éléments de débitage, sont parfaitement interchangeables avec la production bipolaire sur silex fin retrouvée sur le même site de Cheikh Hassan. Il est probable, comme pour les niveaux précédents, que l'on ait affaire aux mêmes tailleurs ou à des tailleurs appartenant à la même tradition pour les deux types de matériaux. Les éléments techniques ainsi que les esquilles d'obsidienne indiquent également que ces tailleurs ont exploité directement sur le site leurs matières premières brutes ou à peine préformées. La production complète des nucléus n'a pas été retrouvée. Cette situation et la rareté même des éléments en obsidienne ne permettent pas de préciser le type de production bipolaire qui est en cause. Signalons une pointe de projectile (fig. 6 , $\left.n^{\circ} 1\right)$ typiquement mureybétienne avec cependant, en position inhabituelle, des retouches sur le corps de la lame dans le but probable d'éliminer un

19. Coqueugniot 1994.

20. On note comme autre différence la présence à Dja'de de pièces esquillées.

21. PERNiCKa et al. 1997. défaut de débitage : cela laisse entrevoir un recours à des lames de second choix dans l'outillage et l'armement. Il en est d'ailleurs de même pour une pointe en silex (fig. $6, n^{\circ} 5$ ) provenant d'une cache du secteur FK. Rappelons que sur le silex, un préfaçonnage de lames sur nucléus bipolaire a pu être démontré, ainsi qu'une exploitation de lames en séries parallèles au moins dans la phase terminale d'exploitation des nucléus à lames.

Si on compare cet ensemble à celui du PPNB également ancien de Mureybet et de Dja'de, on constate aussi que l'obsidienne y est rare et que s'y trouvent notamment des flèches, mais à Dja'de il y a un beau débitage de lamelles par pression ${ }^{19}$, absent à Cheikh Hassan ${ }^{20}$.

\section{Détermination de provenance}

Afin de connaître la provenance de l'obsidienne de Cheikh Hassan (transparente ou gris zoné, aucune pièce ne paraissant verdâtre), sept échantillons ont été analysés (20\% de l'obsidienne de ces deux secteurs, soit $15 \%$ de l'ensemble de la fouille). Ils proviennent tous en fait du secteur atelier : deux pièces ont été étudiées par activation neutronique $^{21}$ et cinq par ICP-MS/ICP-AES ${ }^{22}$.

Les résultats indiquent tous la Cappadoce, plus précisément le Göllü Dag Est, comme unique région d'origine. L'absence de sources en Anatolie orientale est peut-être liée à la faiblesse de l'échantillon analysé encore qu'il représente $15 \%$ de l'ensemble des artefacts en obsidienne, $20 \%$ si on ne considère que deux des secteurs de la fouille. Il est vrai qu'au PPNB ancien de Mureybet dix analyses ont été effectuées correspondant à près du tiers des artefacts en obsidienne $(31 \%)$ : seule une d'elles dénote la source de Bingöl sensu stricto. tandis que, comme à Cheikh Hassan, neuf indiquent également l'ensemble du Göllü Dag Est comme source. À Dja'de là aussi, le Göllü Dag Est est la source la plus exploitée mais il y a également, selon les analyses, de l'obsidienne d'Anatolie orientale, même ici sous forme de petits galets provenant de la région de $\mathrm{Bing}$ ö ${ }^{23}$ à laquelle s'ajoute de l'obsidienne du Nemrut Dag sensu stricto. ${ }^{24}$.

22. MARTINETTO 1996.

23. CoQUeugniot 1998, Bellot-Gurlet 1998.

24. PERNICKA et al. 1997. 

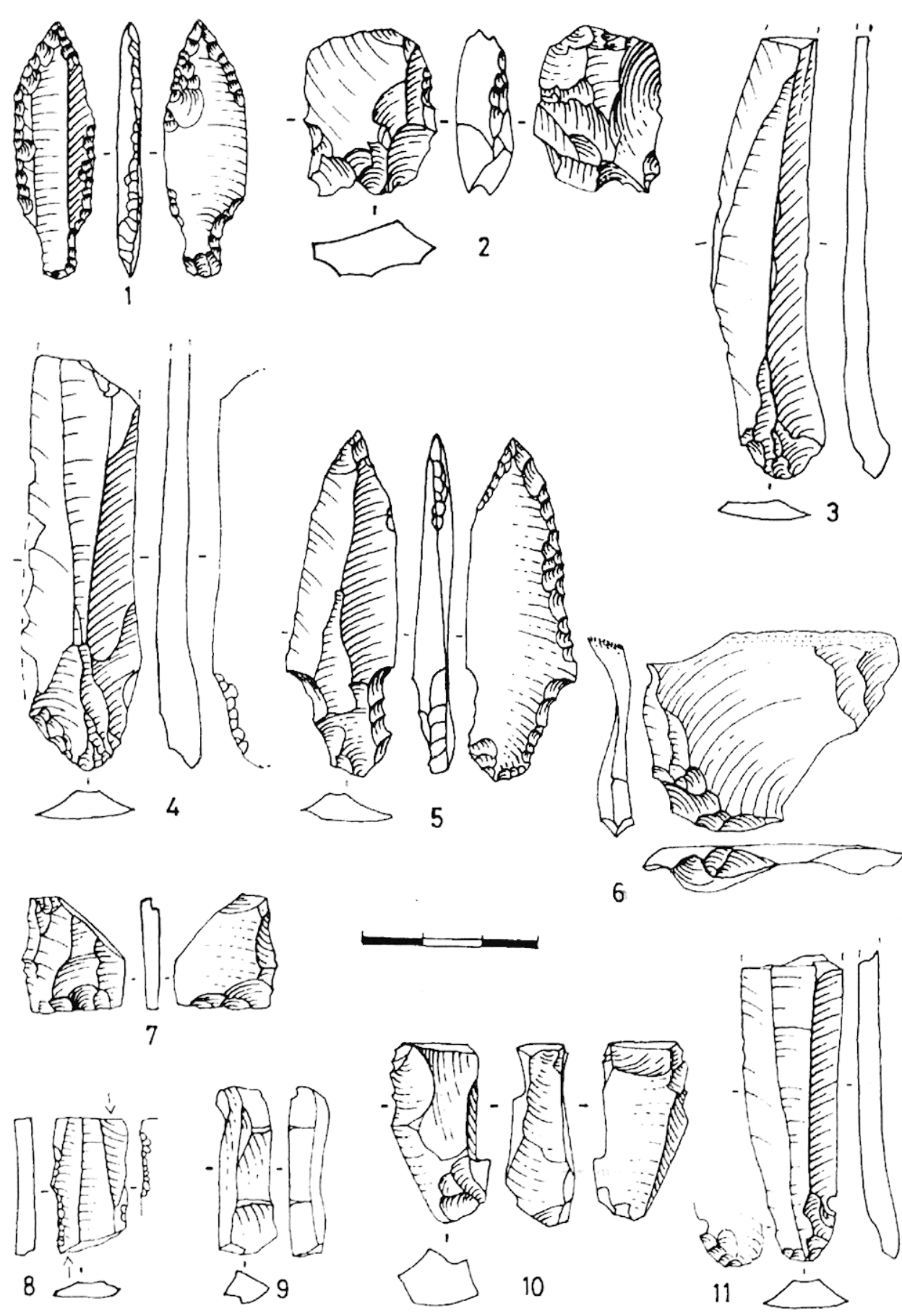

6

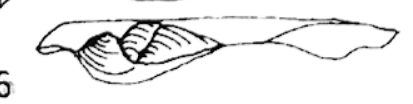

Fig. 6. - Cheikh Hassan : Mobilier PPNB ancien en obsidienne (sauf le ${ }^{\circ} 5$ en silex) provenant du secteur $\mathrm{FK}: \mathrm{n}^{\mathrm{os}} 1-3,5 ;$ du secteur $\mathrm{SF}: \mathrm{n}^{\circ} 4$; et du secteur $\mathrm{JL}: \mathrm{n}^{\mathrm{os}} 6-11$. 


\section{CONCLUSION}

Cette étude nous montre que l'obsidienne est très peu représentée statistiquement par rapport au silex; l'ensemble des pièces retrouvées à Cheikh Hassan, toutes périodes et provenances confondues, ne représenterait même pas la production complète d'un seul nucléus à lames! Son traitement lui-même ne montre pas d'intention particulière par rapport à celui mis en œuvre sur le silex local : ce matériau est débité sur place selon les mêmes modalités que les pièces en silex si ce n'est que les déchets de taille sont ici plus volontiers retouchés, comme par exemple un éclat à crête d'ouverture de plan de frappe, ce qui n'est pas le cas pour le silex. Néanmoins, il n'y a pas de recherche esthétique spéciale le concernant de la part des tailleurs de Cheikh Hassan. Rappelons que le mobilier PPNA ou PPNB est composé d'éclats, de pièces techniques, d'esquilles et de lames qui ont pu être utilisés comme outils avec ou sans retouches. Les pointes de flèches constituent les seules pièces typiques retouchées reconnaissables comme telles.

Toutefois, le caractère exogène de l'obsidienne confère à ces pointes un statut particulier : c'est l'arme en tant que telle, que ce soit au PPNA ou surtout au PPNB, qui est l'élément que l'on valorise. Cette mise en valeur a pu être confirmée par l'analyse spatiale pour unc pointc trouvéc en contexte funéraire au PPNA. Au PPNB, l'unique armature provient du secteur habitat (FK) où elle fut retrouvée directement sur le sol; on a par ailleurs relevé sous le sol d'une des pièces une sépulture comportant un dépôt de lames en silex, tandis que dans l'espace extérieur se trouvait notamment une cache de pointes de projectiles en sile $x^{25}$.

La rareté même de l'obsidienne ne permet pas d'envisager une quelconque importance d'ordre économique dans ce mobilier mais son statut et ses origines lointaines ${ }^{26}$ en font donc un matériau précieux. Celui-ci semble ainsi constituer un bien de prestige intervenant dans un processus d'échange ou de dons au cours de contacts épisodiques entre communautés comme le suggérait Mauss ${ }^{27}$ et ceci se situe entre les sources mêmes

25. ABBiES 1997.

26. Notons que pour l'instant on ne connaît aucun village PPNA dans le voisinage des sources.

27. MAuSs 1923-24. de l'obsidienne et la moyenne vallée de l'Euphrate. Il reste également à préciser quelles voies de circulation ce matériau a pu emprunter, recherches qui sont actuellement en cours.

\section{ABRÉVIATIONS}

\section{Des techniques d'analyses}

AES : Spectrométrie d'émission atomique.

ANRC : Activation avec des neutrons rapides de cyclotron. ICP : Plasma couplé par induction.

LA : Ablation laser.

MS : Spectométrie de masse.

\section{BIBLIOGRAPHIE}

ABBÈs (F.)

1993 « Méthode d'approche de la variabilité laminaire. Application à des armatures perçantes de Cheikh Hassan, Syrie, vIII ${ }^{\mathfrak{e}}$ millénaire B.C. », Cahiers de l'Euphrate 7, p. 119-150.

1997 Étude des industries lithiques du Néolithique précéramique de Syrie du $X^{c}$ au viII mill. B.C. Techniques de débitage et gestion des produits laminaires, thèse de doctorat, université Lumière Lyon 2.

Sous presse «Étude du mobilier lithique d'une unité architecturale de Jerf el-Ahmar (Syrie. PPNA final)». In : I. CANEVA (ed.) Beyond tools. Reconsidering Definitions, counting and Interpretation of Lithic Assemblages, Workshop on PPN Chipped Lithic Industries, Venise, 1998.

Balkan-Atli (N.), Binder (D.) et Cauvin (M.-C.)

1999 «Obsidian: Sources, workshops and trade in Central Anatolia ». In : M. ÖZdoĞAN (ed.). Neolithic in Turkey, Arkeoloji ve Sanat Yay, Istanbul, p. 103-115 et 135-145.

\section{BELLOT-GURLet (L.)}

1998 Caractérisation par analyse élémentaire (PIXE et ICP-MS/-AES) d'un verre naturel : l'obsidienne. Applications à l'étude de provenance d'objets archéologiques, thèse de l'université Joseph Fourier, Grenoble I.

CAuvin (J.)

1980 "Le Moyen Euphrate au vil ${ }^{\mathrm{e}}$ millénaire d'après Mureybet et Cheikh Hassan ». In : J. MARGUERON (éd.), Le Moyen Euphrate, zone de contacts et d'échanges. Actes du Colloque de Strasbourg 1977, E.J. Brill, Leyde, p. 21-34.

1994 Naissance des divinités, naissance de l'agriculture. La Révolution des symboles au Nélithique, Éditions du CNRS, Paris. 
Cauvin (M.-C.)

1991 «L'obsidienne au Levant préhistorique: provenance et fonction ", Cahiers de l'Euphrate 5/6, p. $163-190$.

Cauvin (M.-C.), Chataigner (C.)

1998 «Distribution de l'obsidienne dans les sites archéologiques ». In : M.-C. CAUVIN, A. GourgaUd, B. Gratuze, N. Arnaud, G. Poupeau, J.L. Poidevin et C. Chataigner (éd.), L'obsidienne au Proche et Moyen-Orient. Du volcan à l'outil, Lyon, Maison de l'Orient méditerranéen (British Archaeological Reports, International Series 738), Oxford, p. 325-350.

\section{COQUEUGNIOT (E.)}

1994 «L'industrie lithique de Dja'de el Mughara et le début du PPNB sur l'Euphrate syrien (sondages 1991 et 1992) ». In : H.G. GEBEL et S.K. KozLOWSKI (eds.), Neolithic Chipped Stone Industries of the Fertile Crescent, Ex Oriente, Berlin, p. 313-330.

1998 «Dja'de el Mughara », Chronique archéologique en Syrie, II, ministère de la Culture, direction générale des Antiquités et des Musées, p. 125-132.

Gratuze (B.), Barrandon (J.N.), Al-ISA (K.) et Cauvin (M.-C.)

1993 « Non destructive analysis of obsidian artifacts using nuclear techniques: Investigation of provenance of Near Eastern Artifacts », Archaeometry 35/1, p. 11-21.

Gratuze (B.)

1999 "Obsidian characterisation by laser ablation ICPMS and its application to the prehistoric trade in the Mediterranean and the Near East : The sources and distribution of obsidian within the Aegean and Anatolia ", Journal of Archaeological Sciences 26, p. 869-881.

Hours (F.), Aurenche (O.), Cauvin (J.), Cauvin (M.-

C.), Copeland (L.) et Sanlaville (P.)

1994 Atlas des sites du Proche-Orient (14000-7500 B.P.), (Travaux de la Maison de l'Orient, 25), Maison de l'Orient, Lyon.

MARTINETTO (P.)

1996 Sur la provenance de l'obsidienne de quelques sites archéologiques des $I X^{c}$-VIII $r^{c}$ millénaires B.P. de la haute Vallée de l'Euphrate. Une approche par ICP/AES-MS, mémoire de DESS, université de Bordeaux III.
MAuss (M.)

1923-24 « Essai sur le don. Forme et raison de l'échange dans les sociétés archaiqques", Année sociologique, I (repris In : M. Mauss 1960 , Sociologie et anthropologie, Presses universitaires de France, Paris).

PÉlegrin (J.)

1982 "Approche expérimentale de la technique de production lamellaire d'Orville", Studia praehistorica belgica 2, p. 149-158.

PERLÈS (C.)

1977 « Note préliminaire sur un type d'outil particulier du site d'Orville (Indre) », Bulletin de la Société Préhistorique Française 74, Comptes Rendus des Séances Mensuelles $\mathrm{n}^{\circ}$ 5, p. 141-144.

Pernicka (E.), Keller (J.) et Cauvin (M.-C.)

1997 «Obsidian from Anatolian Sources in the Neolithic of the Middle Euphrates Region (Syria) », Paleorient 23/1, p. 113-122.

POIDEVIN (J.-L.)

1998 «Les gisements d'obsidienne de Turquie et Transcaucasie : géologie, géochimie et chronométrie ". In : M.-C. CAUVIN, A. Gourgaud, B. Gratuze, N. Arnaud, G. Poupeau. J.L. Poidevin et C. Chataigner (éd.), L'obsidienne au Proche et Moyen-Orient. Du volcan à l'outil, Lyon, Maison de l'Orient méditerranéen (British Archaeological Reports, International Series 738), Oxford, p. 105-203.

Santallier (D.), Marechal (C.) et Vera (R.)

1997 «Éléments de parure du Néolithique syrien. identification et provenance des matériaux". Revue d'Archéométrie 21, p. 55-65.

STORDEUR (D.)

1999 «Reprise des fouilles à Cheikh Hassan, une campagne de reconnaissance (Colloque International, Aleppo and Silk Road, du 26 au 30 sept 1994)", Annales archéologiques arabes syriennes 43, p. 59-64.

SUN (S.S.), MCDONOUGH (W.F.)

1989 « Chemical and isotopic systematics of oceanic basalts : implications for mantle composition and processes ». In : A.D. SAUNDERS et M.J. NORRY (eds.), Magmatism in the Ocean basins. (Geological Society, Special Publication, 42). Londres, p. 313-345. 